\title{
Some recurrence relations of poly-Cauchy numbers
}

\section{Takao Komatsu*}

Department of Mathematical Sciences, School of Science, Zhejiang Sci-Tech University, Hangzhou 310018, China.

\begin{abstract}
Poly-Cauchy numbers $c_{n}^{(k)}(n \geqslant 0, k \geqslant 1)$ have explicit expressions in terms of the Stirling numbers of the first kind. When the index is negative, there exists a different expression. However, the sequence $\left\{c_{n}^{(-k)}\right\}_{n} \geqslant 0$ seem quite irregular for a fixed integer $k \geqslant 2$. In this paper we establish a certain kind of recurrence relations among the sequence $\left\{\mathrm{c}_{\mathfrak{n}}^{(-k)}\right\}_{\mathfrak{n} \geqslant 0}$, analyzing the structure of poly-Cauchy numbers. We also study those of poly-Cauchy numbers of the second kind, poly-Euler numbers, and poly-Euler numbers of the second kind. Some different proofs are given. As applications, some leaping relations are shown.
\end{abstract}

Keywords: Poly-Cauchy numbers, poly-Euler numbers, recurrence, leaping relations, Vandermonde's determinant.

2010 MSC: 11B75, 11B37, 11B68, 11B73, 05A19, 11C20, $15 \mathrm{~A} 15$.

(C)2019 All rights reserved.

\section{Introduction}

The poly-Cauchy numbers (of the first kind) are defined by ([9])

$$
c_{n}^{(k)}=n ! \underbrace{\int_{0}^{1} \ldots \int_{0}^{1}}_{k}\left(\begin{array}{c}
x_{1} \cdots x_{k} \\
n
\end{array}\right) d x_{1} \ldots d x_{k}, \quad(n \geqslant 0, k \geqslant 1) .
$$

Their generating function is given by ([9, Theorem 2])

$$
\operatorname{Lif}_{k}(\log (1+x))=\sum_{n=0}^{\infty} c_{n}^{(k)} \frac{x^{n}}{n !}
$$

where

$$
\operatorname{Lif}_{k}(z)=\sum_{m=0}^{\infty} \frac{z^{m}}{m !(m+1)^{k}}
$$

is the polylogarithm factorial (or polyfactorial) function. When $k=1, c_{n}=c_{n}^{(1)}$ are the original Cauchy

\footnotetext{
${ }^{*}$ Corresponding author

Email address: komatsu@zstu.edu.cn (Takao Komatsu)

doi: $10.22436 /$ jnsa.012.12.05
}

Received: 2019-06-01 Revised: 2019-07-01 Accepted: 2019-07-23 
numbers, whose generating function is given by $([3,12])$

$$
\frac{x}{\log (1+x)}=\sum_{n=0}^{\infty} c_{n} \frac{x^{n}}{n !} .
$$

In 2018 Sasaki [14] showed several recurrence relations concerning the poly-Bernoulli numbers with negative indices. Poly-Bernoulli numbers $\mathrm{B}_{\mathrm{n}}^{(\mathrm{k})}([7])$ are defined by

$$
\frac{\mathrm{Li}_{k}\left(1-e^{-x}\right)}{1-e^{-x}}=\sum_{n=0}^{\infty} B_{n}^{(k)} \frac{x^{n}}{n !}
$$

where

$$
\mathrm{Li}_{\mathrm{k}}(z)=\sum_{\mathrm{m}=1}^{\infty} \frac{z^{\mathrm{m}}}{\mathrm{m}^{\mathrm{k}}}
$$

is the polylogarithm function. When $k=1, B_{n}=B_{n}^{(1)}$ are the classical Bernoulli numbers with $B_{1}^{(1)}=1 / 2$, whose generating function is given by

$$
\frac{x}{1-e^{-x}}=\sum_{n=0}^{\infty} B_{n} \frac{x^{n}}{n !} .
$$

Poly-Bernoulli numbers $\mathrm{B}_{n}^{(k)}$ have explicit forms in terms of the Stirling numbers of the second kind ([7]):

$$
B_{n}^{(k)}=\sum_{m=0}^{n}\left\{\begin{array}{c}
n \\
m
\end{array}\right\} \frac{(-1)^{n-m} m !}{(m+1)^{k}} .
$$

If the index is negative, another form is given by ([2, Theorem 2])

$$
B_{n}^{(-k)}=\sum_{j=0}^{\min (n, k)}(j !)^{2}\left\{\begin{array}{l}
n+1 \\
j+1
\end{array}\right\}\left\{\begin{array}{l}
k+1 \\
j+1
\end{array}\right\} .
$$

Many relations and identities for Bernoulli numbers have been known and several ones for polyBernoulli numbers have been discovered. Sasaki has found some new relations for poly-Bernoulli numbers together with Ohno. The main result is stated as

$$
\sum_{0 \leqslant i \leqslant j \leqslant m}(-1)^{i}\left[\begin{array}{c}
m+2 \\
i+1
\end{array}\right] B_{n+j}^{(-k)}=0 .
$$

By using the falling factorial, we determine the coefficients $s_{i}^{(n)}$ by

$$
(x-2)_{n}=s_{n}^{(n)} x^{n}+s_{n-1}^{(n)} x^{n-1}+\cdots+s_{1}^{(n)} x+s_{0}^{(n)} .
$$

Each coefficient $s_{n-l}^{(n)}(j=0,1, \ldots, n)$ can be written in terms of the (unsigned) Stirling numbers of the first kind as

$$
s_{n-l}^{(n)}=\sum_{j=0}^{l}(-1)^{j}\left[\begin{array}{c}
n+2 \\
n+2-j
\end{array}\right] .
$$

Then, (1.1) is equivalent to the following convenient form.

Lemma 1.1. For $n \geqslant k$ and $m \geqslant k$,

$$
\sum_{j=0}^{m} s_{j}^{(m)} B_{n-m+j}^{(-k)}=0 .
$$


For example, by $(x-2)_{2}=x^{2}-5 x+6$, we have

$$
\mathrm{B}_{\mathrm{n}}^{(-2)}=5 \mathrm{~B}_{\mathrm{n}-1}^{(-2)}-6 \mathrm{~B}_{\mathrm{n}-2}^{(-2)} \text {. }
$$

By $(x-2)_{3}=x^{3}-9 x^{2}+26 x-24$, we have

$$
\mathrm{B}_{\mathrm{n}}^{(-3)}=9 \mathrm{~B}_{\mathrm{n}-1}^{(-3)}-26 \mathrm{~B}_{\mathrm{n}-2}^{(-3)}+24 \mathrm{~B}_{\mathrm{n}-3}^{(-3)} \text {. }
$$

In fact, $B_{n}^{(-2)}=2 \cdot 3^{n}-2^{n}([15, A 027649])$ and $B_{n}^{(-3)}=2^{n}-2 \cdot 3^{n+1}+6 \cdot 4^{n}([15, A 027650])$.

Poly-Cauchy numbers (of the first kind) have an explicit form in terms of the (unsigned) Stirling numbers of the first kind ([9, Theorem 1]):

$$
c_{n}^{(k)}=\sum_{m=0}^{n}\left[\begin{array}{l}
n \\
m
\end{array}\right] \frac{(-1)^{n-m}}{(m+1)^{k}}, \quad(n \geqslant 0, k \geqslant 1) .
$$

The definition may restrict the index $k$ as nonnegative values, but the above expression is possible for negative $k$ too. If the index is negative, another form is given by ([5, Theorem 8 Remark $]$ )

$$
c_{n}^{(-k)}=\sum_{j=0}^{k}(-1)^{n+j} j !\left(\left[\begin{array}{l}
n \\
j
\end{array}\right]-n\left[\begin{array}{c}
n-1 \\
j
\end{array}\right]\right)\left\{\begin{array}{l}
k+1 \\
j+1
\end{array}\right\}, \quad(n \geqslant 0, k \geqslant 0) .
$$

It is easy to see that

$$
c_{n}^{(-1)}=(-1)^{n}(n-2) ! \quad(n \geqslant 2) .
$$

In [6], some congruence relations of poly-Cauchy numbers $c_{n}^{(-k)}$ are investigated by $k$ being fixed for all sufficiently large $n$. However, more precise regularity of the following sequences ([15, A222627, A222636, A222748, A223023]) has not been found:

$$
\begin{aligned}
& \left\{\mathfrak{c}_{\mathfrak{n}}^{(-2)}\right\}_{\mathfrak{n} \geqslant 0}=1,4,5,-3,4,-8,20,-52,72,936,-17568,238752,-3113280, \ldots, \\
& \left\{\mathfrak{c}_{\mathfrak{n}}^{(-3)}\right\}_{\mathfrak{n} \geqslant 0}=1,8,19,-1,-10,48,-234,1302,-8328,60672,-497688, \ldots, \\
& \left\{\mathfrak{c}_{\mathfrak{n}}^{(-4)}\right\}_{\mathfrak{n} \geqslant 0}=1,16,65,45,-116,340,-1240,5480,-28464,169248, \ldots, \\
& \left\{\mathfrak{c}_{\mathfrak{n}}^{(-5)}\right\}_{\mathfrak{n} \geqslant 0}=1,32,211,359,-538,984,-1866,1110,32640,-449760, \ldots
\end{aligned}
$$

In this paper we establish a certain kind of recurrence relations among the sequence $\left\{\mathbf{c}_{n}^{(-k)}\right\}_{\mathfrak{n} \geqslant 0}$, analyzing the structure of poly-Cauchy numbers. We also study those of poly-Cauchy numbers of the second kind, poly-Euler numbers and poly-Euler numbers of the second kind. Some different proofs are given. As applications, some leaping relations are shown.

\section{Poly-Cauchy numbers with negative indices}

Now, we are ready to present our main theorem, which can explain how the sequences $\left\{c_{\mathfrak{n}}^{(-k)}\right\}_{\mathfrak{n} \geqslant 0}$ are generated, though they do not seem to have any clear regularity.

Theorem 2.1. For $n \geqslant k+2$,

$$
c_{n}^{(-k)}+\sum_{l=1}^{k}\left(\sum_{l+1 \leqslant i_{1} \leqslant \cdots \leqslant i_{l} \leqslant k+1}\left(n-i_{1}\right) \cdots\left(n-i_{l}\right)\right) c_{n-l}^{(-k)}=0 .
$$

Remark 2.2. When $k=1,2,3$, we have

$$
\begin{aligned}
& c_{n}^{(-1)}+(n-2) c_{n-1}^{(-1)}=0 \quad(n \geqslant 3), \\
& c_{n}^{(-2)}+(n-2+n-3) c_{n-1}^{(-2)}+(n-3)^{2} c_{n-2}^{(-2)}=0 \quad(n \geqslant 4),
\end{aligned}
$$




$$
\begin{aligned}
& c_{n}^{(-3)}+(n-2+n-3+n-4) c_{n-1}^{(-3)} \\
& \quad+\left((n-3)^{2}+(n-3)(n-4)+(n-4)^{2}\right) c_{n-2}^{(-3)}+(n-4)^{2} c_{n-3}^{(-3)}=0 \quad(n \geqslant 5)
\end{aligned}
$$

respectively.

Proof of Theorem 2.1. For a fixed integer $k \geqslant 1$, assume that the numbers $c_{n}^{(-k)}$ satisfy the recurrence relation

$$
c_{n}^{(-k)}+a_{1, n} c_{n-1}^{(-k)}+a_{2, n} c_{n-2}^{(-k)}+\cdots+a_{k, n} c_{n-k}^{(-k)}=0 .
$$

By the recurrence relation

$$
\left[\begin{array}{c}
n+1 \\
k
\end{array}\right]=n\left[\begin{array}{l}
n \\
k
\end{array}\right]+\left[\begin{array}{c}
n \\
k-1
\end{array}\right] \text { with }\left[\begin{array}{l}
n \\
0
\end{array}\right]=0 \quad(n \geqslant 1)
$$

the form (1.2) can be also written as

$$
c_{n}^{(-k)}=\sum_{j=0}^{k}(-1)^{n+j+1} j !\left(\left[\begin{array}{c}
n-1 \\
j
\end{array}\right]-\left[\begin{array}{c}
n-1 \\
j-1
\end{array}\right]\right)\left\{\begin{array}{l}
k+1 \\
j+1
\end{array}\right\} \quad(n \geqslant 0, k \geqslant 0) .
$$

Here, one determines [1, Table 2.2] that

$$
\left[\begin{array}{c}
-n^{\prime} \\
k
\end{array}\right]= \begin{cases}1, & \text { if } n^{\prime} \geqslant 0, k=-n^{\prime} \\
0, & \text { if } n^{\prime} \geqslant 0, k \geqslant-n^{\prime}+1\end{cases}
$$

Since the relation (2.1) does not depend on the values $\left\{\begin{array}{l}k+1 \\ j+1\end{array}\right\}$, we have the system of equations

$$
\begin{aligned}
& -\left[\begin{array}{c}
n-1 \\
1
\end{array}\right]+\left[\begin{array}{c}
n-2 \\
1
\end{array}\right] a_{1, n}-\left[\begin{array}{c}
n-3 \\
1
\end{array}\right] a_{2, n}+\cdots+(-1)^{k+1}\left[\begin{array}{c}
n-k-1 \\
1
\end{array}\right] a_{k, n}=0, \\
& -\left(\left[\begin{array}{c}
n-1 \\
2
\end{array}\right]-\left[\begin{array}{c}
n-1 \\
1
\end{array}\right]\right)+\left(\left[\begin{array}{c}
n-2 \\
2
\end{array}\right]-\left[\begin{array}{c}
n-2 \\
1
\end{array}\right]\right) a_{1, n} \\
& -\left(\left[\begin{array}{c}
n-3 \\
2
\end{array}\right]-\left[\begin{array}{c}
n-3 \\
1
\end{array}\right]\right) a_{2, n}+\cdots+(-1)^{k+1}\left(\left[\begin{array}{c}
n-k-1 \\
2
\end{array}\right]-\left[\begin{array}{c}
n-k-1 \\
1
\end{array}\right]\right) a_{k, n}=0, \\
& -\left(\left[\begin{array}{c}
n-1 \\
3
\end{array}\right]-\left[\begin{array}{c}
n-1 \\
2
\end{array}\right]\right)+\left(\left[\begin{array}{c}
n-2 \\
3
\end{array}\right]-\left[\begin{array}{c}
n-2 \\
2
\end{array}\right]\right) a_{1, n} \\
& -\left(\left[\begin{array}{c}
n-3 \\
3
\end{array}\right]-\left[\begin{array}{c}
n-3 \\
2
\end{array}\right]\right) a_{2, n}+\cdots+(-1)^{k+1}\left(\left[\begin{array}{c}
n-k-1 \\
3
\end{array}\right]-\left[\begin{array}{c}
n-k-1 \\
2
\end{array}\right]\right) a_{k, n}=0, \\
& -\left(\left[\begin{array}{c}
n-1 \\
k
\end{array}\right]-\left[\begin{array}{c}
n-1 \\
k-1
\end{array}\right]\right)+\left(\left[\begin{array}{c}
n-2 \\
k
\end{array}\right]-\left[\begin{array}{c}
n-2 \\
k-1
\end{array}\right]\right) a_{1, n} \\
& -\left(\left[\begin{array}{c}
n-3 \\
k
\end{array}\right]-\left[\begin{array}{c}
n-3 \\
k-1
\end{array}\right]\right) a_{2, n}+\cdots+(-1)^{k+1}\left(\left[\begin{array}{c}
n-k-1 \\
k
\end{array}\right]-\left[\begin{array}{c}
n-k-1 \\
k-1
\end{array}\right]\right) a_{k, n}=0,
\end{aligned}
$$

which is equivalent to the system

$$
\begin{aligned}
& -\left[\begin{array}{c}
n-1 \\
1
\end{array}\right]+\left[\begin{array}{c}
n-2 \\
1
\end{array}\right] a_{1, n}-\left[\begin{array}{c}
n-3 \\
1
\end{array}\right] a_{2, n}+\cdots+(-1)^{k+1}\left[\begin{array}{c}
n-k-1 \\
1
\end{array}\right] a_{k, n}=0, \\
& -\left[\begin{array}{c}
n-1 \\
2
\end{array}\right]+\left[\begin{array}{c}
n-2 \\
2
\end{array}\right] a_{1, n}-\left[\begin{array}{c}
n-3 \\
2
\end{array}\right] a_{2, n}+\cdots+(-1)^{k+1}\left[\begin{array}{c}
n-k-1 \\
2
\end{array}\right] a_{k, n}=0, \\
& -\left[\begin{array}{c}
n-1 \\
3
\end{array}\right]+\left[\begin{array}{c}
n-2 \\
3
\end{array}\right] a_{1, n}-\left[\begin{array}{c}
n-3 \\
3
\end{array}\right] a_{2, n}+\cdots+(-1)^{k+1}\left[\begin{array}{c}
n-k-1 \\
3
\end{array}\right] a_{k, n}=0,
\end{aligned}
$$




$$
-\left[\begin{array}{c}
n-1 \\
k
\end{array}\right]+\left[\begin{array}{c}
n-2 \\
k
\end{array}\right] a_{1, n}-\left[\begin{array}{c}
n-3 \\
k
\end{array}\right] a_{2, n}+\cdots+(-1)^{k+1}\left[\begin{array}{c}
n-k-1 \\
k
\end{array}\right] a_{k, n}=0 .
$$

By Cramer, we have

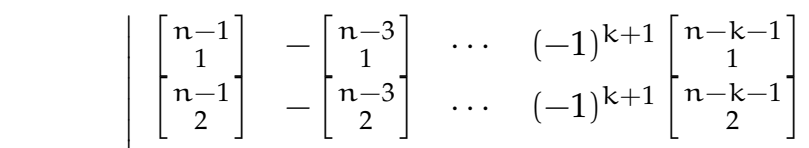

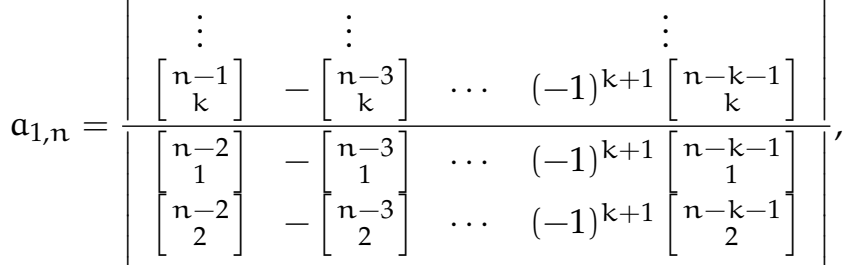

$$
\begin{aligned}
& {\left[\begin{array}{cccc}
\vdots & \vdots & & \vdots \\
{\left[\begin{array}{c}
n-2 \\
k
\end{array}\right]} & -\left[\begin{array}{c}
n-3 \\
k
\end{array}\right] & \cdots & (-1)^{k+1}\left[\begin{array}{c}
n-k-1 \\
k
\end{array}\right]
\end{array}\right.}
\end{aligned}
$$

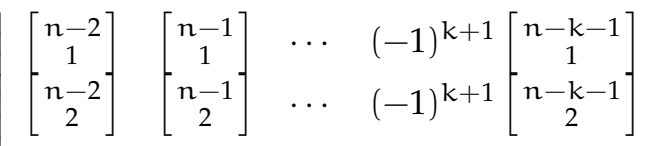

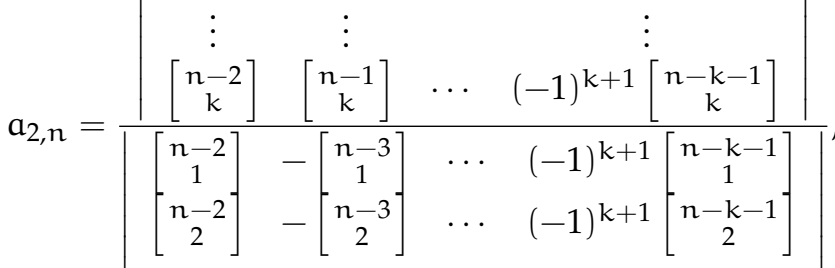

$$
\begin{aligned}
& \begin{array}{cccc}
\vdots & \vdots & & \vdots \\
{\left[\begin{array}{c}
n-2 \\
k
\end{array}\right]} & -\left[\begin{array}{c}
n-3 \\
k
\end{array}\right] & \cdots & (-1)^{k+1}\left[\begin{array}{c}
n-k-1 \\
k
\end{array}\right]
\end{array} \\
& \text { : }
\end{aligned}
$$

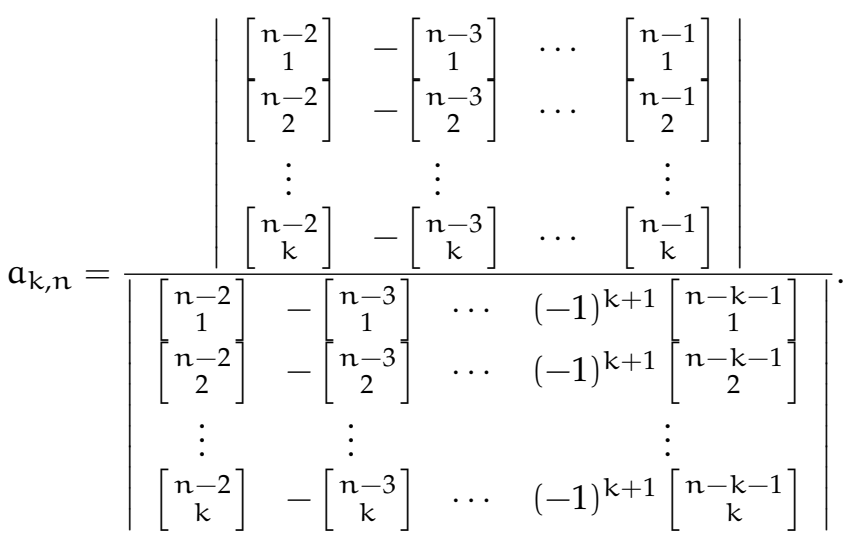

First, we show that the denominator

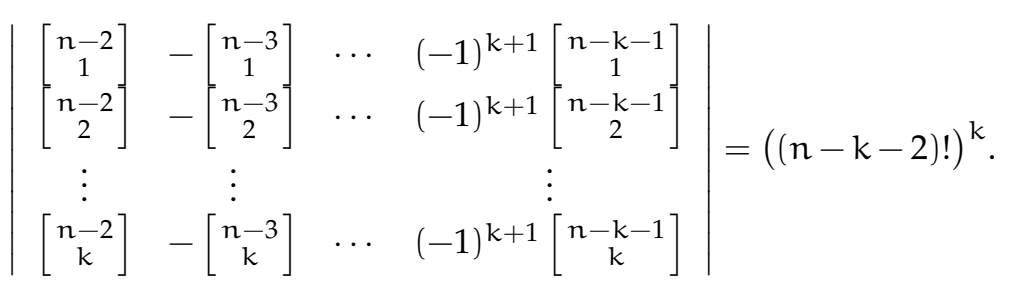

By doing the operation: the 1 st column $+(n-3) \times$ the 2 nd column, the 2 nd column $+(n-4) \times$ the 3 rd 
column, $\ldots$, the $(k-1)$ th column $+(n-k-1) \times$ the $k$ th column, using the recurrence relation $(2.2)$, the left-hand side of (2.3) is equal to

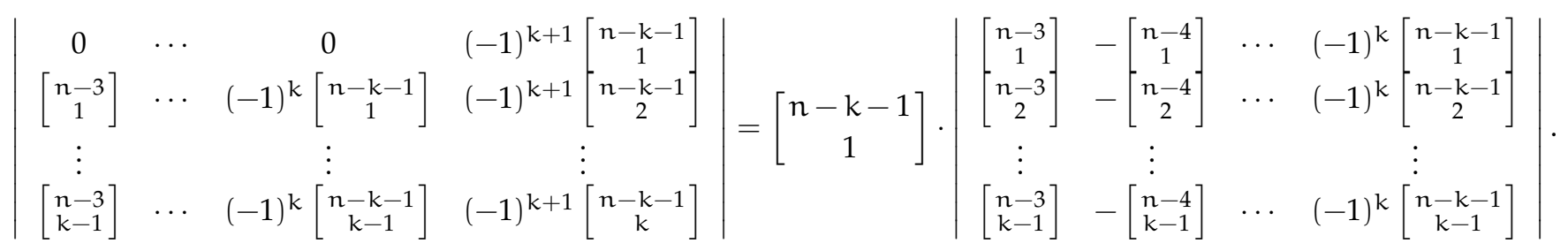

Repeating the similar steps, the left-hand side of (2.3) is equal to

$$
\begin{aligned}
& \left(\left[\begin{array}{c}
n-k-1 \\
1
\end{array}\right]\right)^{2} \cdot\left|\begin{array}{cccc}
{\left[\begin{array}{c}
n-4 \\
1
\end{array}\right]} & -\left[\begin{array}{c}
n-5 \\
1
\end{array}\right] & \cdots & (-1)^{k-1}\left[\begin{array}{c}
n-k-1 \\
1 \\
2
\end{array}\right] \\
\vdots & -\left[\begin{array}{c}
n-5 \\
2
\end{array}\right] & \cdots & (-1)^{k-1}\left[\begin{array}{c}
n-k-1 \\
2
\end{array}\right] \\
\vdots & & \vdots \\
{\left[\begin{array}{c}
n-4 \\
k-2
\end{array}\right]} & -\left[\begin{array}{c}
n-5 \\
k-2
\end{array}\right] & \cdots & (-1)^{k-1}\left[\begin{array}{c}
n-k-1 \\
k-2
\end{array}\right]
\end{array}\right|
\end{aligned}
$$

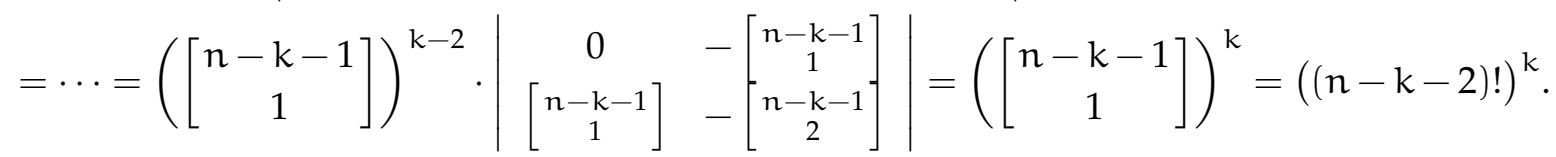

Now, we can write the numerators of $a_{j, n}(j=1,2, \ldots, k)$ as

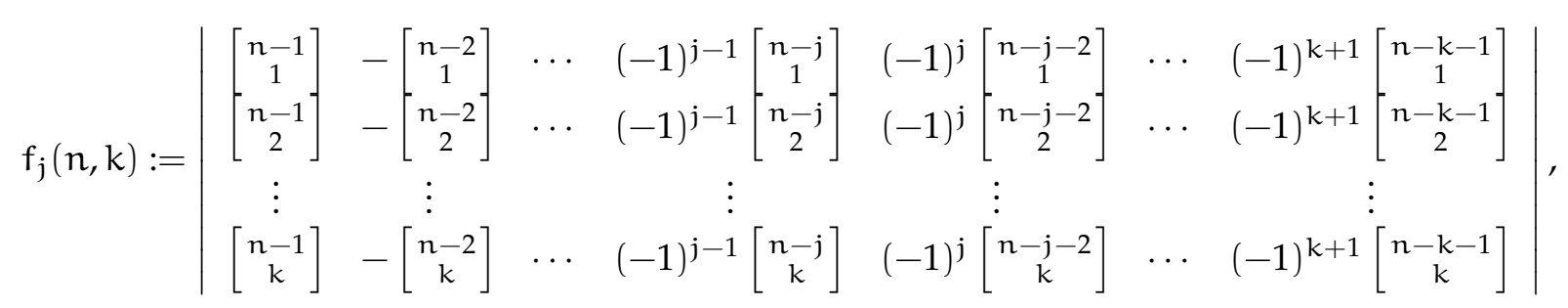

where there exists a gap without $\left[\begin{array}{c}n-j-1 \\ \ell\end{array}\right](1 \leqslant \ell \leqslant k)$. In this sense, we can put the denominator (2.3) as $f_{0}(n, k)$. We shall show that $f_{j}(n, k)$ has the recursion formula

$$
f_{j}(n, k)=(n-k-2) !\left((n-j-1) f_{j-1}(n-1, k-1)+f_{j}(n-1, k-1)\right) \quad(n \geqslant k+2),
$$

with $f_{0}(n, k)=((n-k-2) !)^{k}$ and $f_{k}(n, k)=((n-k-1) !)^{k}(k \geqslant 0)$. We do the following operations:

1. the 1 st column $+(n-2) \times$ the 2 nd column, the 2 nd column $+(n-3) \times$ the 3 rd column, $\ldots$, the $(j-1)$ th column $+(n-j) \times$ the $j$ th column;

2. the determinant is divided into two parts by applying the recurrence relation $(2.2)$ on the $(j-1)$ th column;

3. for the first determinant the $j$ th column $+(n-j-2) \times$ the $(j+1)$ th column, the $(j+1)$ th column $+(n-$ $j-3) \times(j+2)$ th column, $\ldots$, the $(k-1)$ th column $+(n-k-1) \times$ the $k$ th column, for the second determinant the $(j+1)$ the column $+(n-j-3) \times(j+2)$ th column, $\ldots$, the $(k-1)$ th column $+(n-$ $k-1) \times$ the kth column.

Therefore, we have

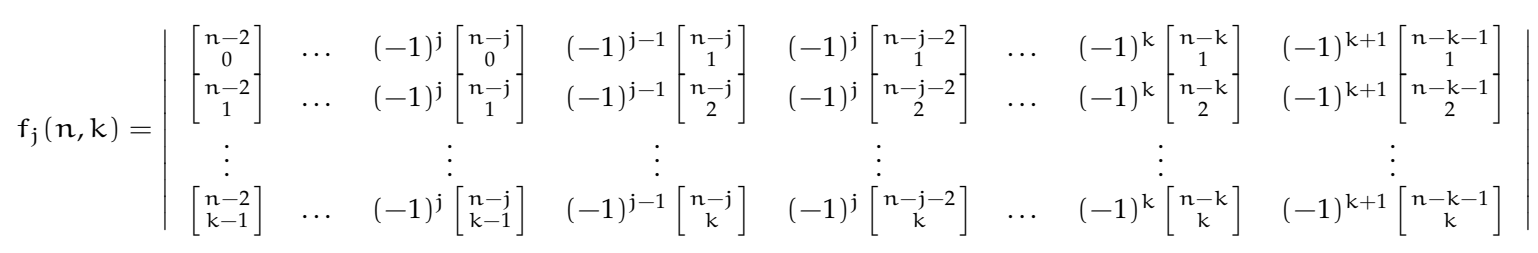




$$
\begin{aligned}
& =(n-j-1)
\end{aligned}
$$

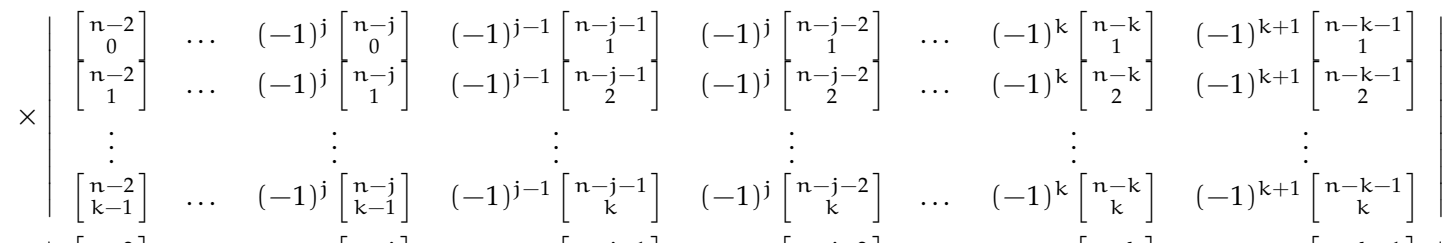

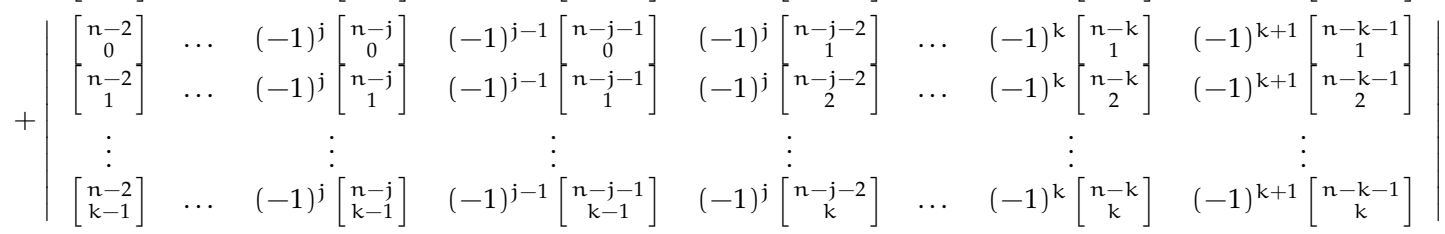

$$
\begin{aligned}
& =(n-j-1)
\end{aligned}
$$

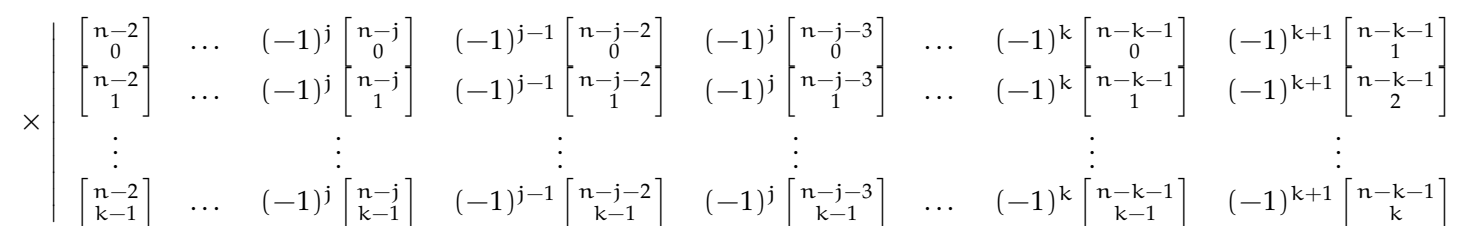

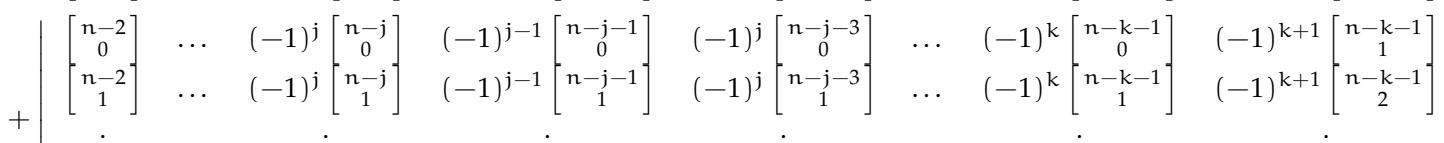

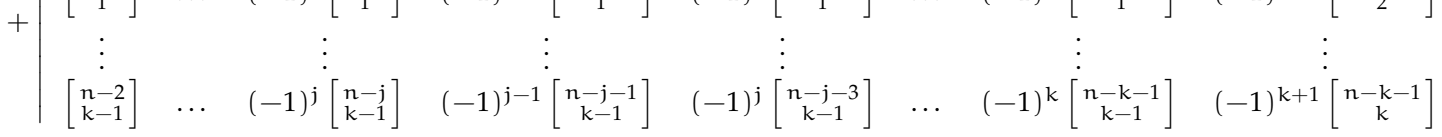

$$
\begin{aligned}
& =(n-k-2) !(n-j-1) f_{j-1}(n-1, k-1)+(n-k-2) ! f_{j}(n-1, k-1) \text {, }
\end{aligned}
$$

which is (2.4).

Now, by induction, we can show that

$$
f_{j}(n, k)=((n-k-2) !)^{k} \sum_{j+1 \leqslant i_{1} \leqslant \cdots \leqslant i_{j} \leqslant k+1}\left(n-i_{1}\right) \cdots\left(n-i_{j}\right) .
$$

The result is valid for $j=0$, and is clear for $k=1,2$. Assume that the result is valid up to $j-1$. Then by (2.4)

$$
\begin{aligned}
f_{j}(n, k)= & (n-k-2) !(n-j-1)((n-k-2) !)^{k-1} \sum_{j \leqslant i_{1} \leqslant \cdots \leqslant i_{j-1} \leqslant k}\left(n-i_{1}-1\right) \cdots\left(n-i_{j-1}-1\right) \\
& +(n-k-2) ! f_{j}(n-1, k-1) .
\end{aligned}
$$

Repeating the similar steps, we have

$$
\begin{aligned}
f_{j}(n, k)= & ((n-k-2) !)^{k}(n-j-1) \sum_{j \leqslant i_{1} \leqslant \cdots \leqslant i_{j-1} \leqslant k}\left(n-i_{1}-1\right) \cdots\left(n-i_{j-1}-1\right) \\
& +((n-k-2) !)^{k}(n-j-2) \sum_{j \leqslant i_{1} \leqslant \cdots \leqslant i_{j-1} \leqslant k-1}\left(n-i_{1}-2\right) \cdots\left(n-i_{j-1}-2\right) \\
& +((n-k-2) !)^{2} f_{j}(n-2, k-2) \\
= & ((n-k-2) !)^{k}(n-j-1) \sum_{j \leqslant i_{1} \leqslant \cdots \leqslant i_{j-1} \leqslant k}\left(n-i_{1}-1\right) \cdots\left(n-i_{j-1}-1\right) \\
& +((n-k-2) !)^{k}(n-j-2) \sum_{j \leqslant i_{1} \leqslant \cdots \leqslant i_{j-1} \leqslant k-1}\left(n-i_{1}-2\right) \cdots\left(n-i_{j-1}-2\right) \\
& \vdots \\
& +((n-k-2) !)^{k}(n-k) \sum_{j \leqslant i_{1} \leqslant \cdots \leqslant i_{j-1} \leqslant j+1}\left(n-i_{1}+j-k\right) \cdots\left(n-i_{j-1}+j-k\right)
\end{aligned}
$$




$$
+((n-k-2) !)^{k-j} f_{j}(n-k+j, j)
$$

where

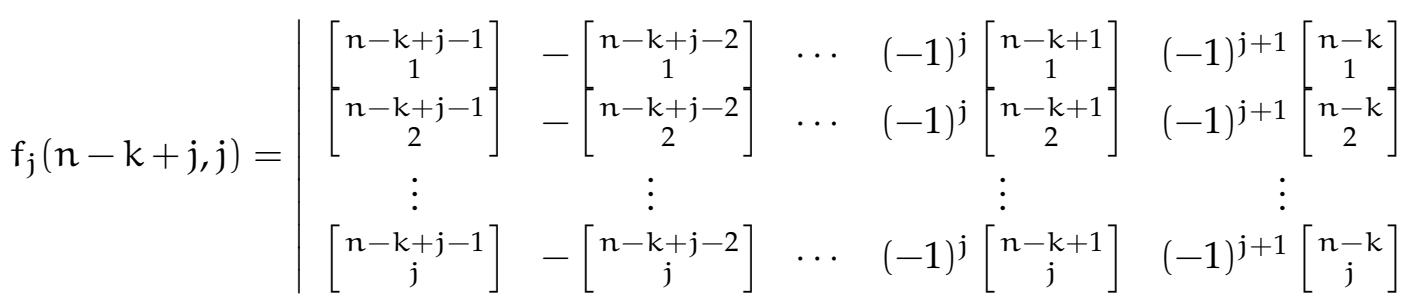

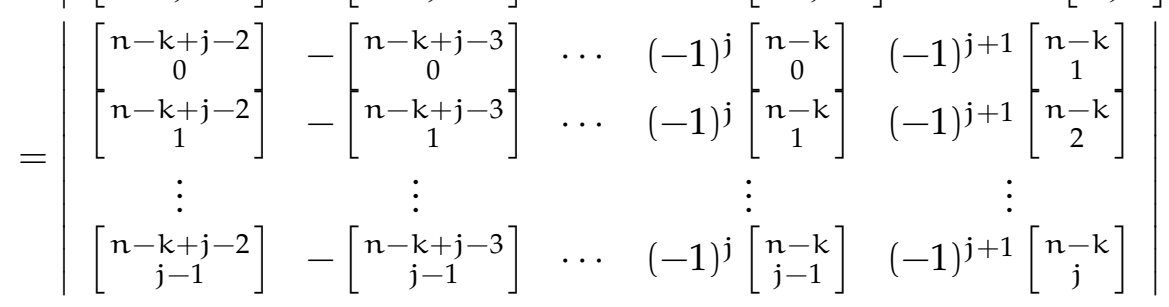

$$
\begin{aligned}
& =\left[\begin{array}{c}
n-k \\
1
\end{array}\right] \cdot\left|\begin{array}{ccc}
{\left[\begin{array}{c}
n-k+j-2 \\
1
\end{array}\right]} & \cdots & (-1)^{j}\left[\begin{array}{c}
n-k \\
1
\end{array}\right] \\
\vdots & & \vdots \\
{\left[\begin{array}{c}
n-k+j-2 \\
j-1
\end{array}\right]} & \cdots & (-1)^{j}\left[\begin{array}{c}
n-k \\
j-1
\end{array}\right]
\end{array}\right| \\
& =\left(\left[\begin{array}{c}
n-k \\
1
\end{array}\right]\right)^{j-2}\left|\left[\begin{array}{c}
n-k+1 \\
1 \\
n-k+1 \\
2
\end{array}\right]-\left[\begin{array}{c}
n-k \\
1 \\
2
\end{array}\right]\right| \\
& =\left(\left[\begin{array}{c}
n-k \\
1
\end{array}\right]\right)^{j-2}\left|\left[\begin{array}{cc}
n-k \\
0 \\
n-k \\
1
\end{array}\right]-\left[\begin{array}{c}
n-k \\
1 \\
2
\end{array}\right]\right| \\
& =\left(\left[\begin{array}{c}
n-k \\
1
\end{array}\right]\right)^{j}=((n-k-1) !)^{j} \text {. }
\end{aligned}
$$

Thus, we obtain the formula (2.5).

\section{Poly-Cauchy numbers of the second kind}

The poly-Cauchy numbers of the second kind are defined by ([9])

$$
\widehat{c}_{\mathrm{n}}^{(\mathrm{k})}=\mathrm{n} ! \underbrace{\int_{0}^{1} \ldots \int_{0}^{1}}_{\mathrm{k}}\left(\begin{array}{c}
-\mathrm{x}_{1} \cdots \mathrm{x}_{\mathrm{k}} \\
\mathrm{n}
\end{array}\right) \mathrm{d} x_{1} \ldots \mathrm{d} \mathrm{x}_{\mathrm{k}} .
$$

Their generating function is given by ([9, Theorem 5])

$$
\operatorname{Lif}_{k}(-\log (1+x))=\sum_{n=0}^{\infty} \widehat{c}_{n}^{(k)} \frac{x^{n}}{n !} .
$$

When $\mathrm{k}=1, \widehat{\mathrm{c}}_{\mathrm{n}}=\widehat{\mathrm{c}}_{\mathrm{n}}^{(1)}$ are the original Cauchy numbers of the second kind, whose generating function is given by

$$
\frac{x}{(1+x) \log (1+x)}=\sum_{n=0}^{\infty} \widehat{c}_{n} \frac{x^{n}}{n !}
$$


Poly-Cauchy numbers of the second kind have an explicit form in terms of the (unsigned) Stirling numbers of the first kind ([9, Theorem 4]):

$$
\widehat{\mathrm{c}}_{n}^{(\mathrm{k})}=(-1)^{\mathrm{n}} \sum_{m=0}^{\mathrm{n}}\left[\begin{array}{l}
n \\
m
\end{array}\right] \frac{1}{(m+1)^{k}} .
$$

If the index is negative, poly-Cauchy numbers of the second kind have a different expression ([5, Theorem 8 Remark]):

$$
\widehat{c}_{n}^{(-k)}=\sum_{j=0}^{k}(-1)^{n} j !\left[\begin{array}{c}
n+1 \\
j+1
\end{array}\right]\left\{\begin{array}{l}
k+1 \\
j+1
\end{array}\right\} .
$$

Similarly to Theorem 2.1, poly-Cauchy numbers of the second kind with negative indices have the following recurrence relations.

Theorem 3.1. For $\mathrm{n} \geqslant k+1$,

$$
\widehat{\mathbf{c}}_{n}^{(-k)}+\sum_{l=1}^{k+1}\left(\sum_{l-1 \leqslant i_{1} \leqslant \cdots \leqslant i_{l} \leqslant k}\left(n-i_{1}\right) \cdots\left(n-i_{l}\right)\right) \widehat{c}_{n-l}^{(-k)}=0 .
$$

Remark 3.2. When $\mathrm{k}=0,1,2$, we have

$$
\begin{aligned}
& \widehat{\mathbf{c}}_{n}^{(0)}+n \widehat{\mathbf{c}}_{n-1}^{(0)}=0 \quad(n \geqslant 1), \\
& \widehat{\mathbf{c}}_{n}^{(-1)}+(n+n-1) \widehat{\mathbf{c}}_{n-1}^{(-1)}+(n-1)^{2} \widehat{\mathbf{c}}_{n-2}^{(-1)}=0 \quad(n \geqslant 2), \\
& \widehat{\mathbf{c}}_{n}^{(-2)}+(n+n-1+n-2) \widehat{\mathbf{c}}_{n-1}^{(-2)} \\
& \quad+\left((n-1)^{2}+(n-1)(n-2)+(n-2)^{2}\right) \widehat{c}_{n-2}^{(-2)}+(n-2)^{3} \widehat{c}_{n-2}^{(-2)}=0 \quad(n \geqslant 3),
\end{aligned}
$$

respectively. Since

$$
\widehat{\mathbf{c}}_{n}^{(0)}=(-1)^{n} \sum_{k=0}^{n}\left[\begin{array}{l}
n \\
k
\end{array}\right]=(-1)^{n} n !
$$

the first relation is clear.

\section{Poly-Euler numbers}

For an integer $k$, poly-Euler numbers $E_{n}^{(k)}(n \geqslant 0)$ are defined by $([13])$

$$
\frac{\operatorname{Li}_{k}\left(1-e^{-4 x}\right)}{4 x \cosh x}=\sum_{n=0}^{\infty} E_{n}^{(k)} \frac{x^{n}}{n !} .
$$

When $\mathrm{k}=1, \mathrm{E}_{\mathrm{n}}=\mathrm{E}_{\mathrm{n}}^{(1)}$ are the classical Euler numbers, defined by

$$
\frac{1}{\cosh x}=\sum_{n=0}^{\infty} E_{n} \frac{x^{n}}{n !}
$$

Poly-Euler numbers can be expressed in terms of poly-Bernoulli numbers ([13, Theorem 2.1]):

$$
E_{n}^{(k)}=\frac{1}{2(n+1)} \sum_{m=0}^{n+1}\left(\begin{array}{c}
n+1 \\
m
\end{array}\right) 4^{m}\left((-1)^{n-m+1}-(-3)^{n-m+1}\right) B_{m}^{(k)} .
$$


In the case of negative index, poly-Euler numbers have an explicit expression ([13, Theorem 6.1]):

$$
(n+1) E_{n}^{(-k)}=\frac{(-1)^{k}}{2} \sum_{l=0}^{k}(-1)^{l} l !\left\{\begin{array}{l}
k \\
l
\end{array}\right\}\left((4 l+3)^{n+1}-(4 l+1)^{n+1}\right) \quad(k \geqslant 0) .
$$

Notice that $E_{n}^{(-k)}$ are not necessarily integers but $(n+1) E_{n}^{(-k)}$ are all integers. For convenience, put $\widetilde{E}_{n}^{(-k)}=(n+1) E_{n}^{(-k)}$. By using the generalized falling factorial, determine the coefficients $\sigma_{i}^{(n)}$ by

$$
(x-5 \mid 2)_{n}=(x-5)(x-7) \cdots(x-2 n-3)=\sigma_{n}^{(n)} x^{n}+\sigma_{n-1}^{(n)} x^{n-1}+\cdots+\sigma_{1}^{(n)} x+\sigma_{0}^{(n)} .
$$

Then Euler numbers with negative indices satisfy the following recurrence formula.

Theorem 4.1. For $\mathrm{k}=0$,

$$
\widetilde{\mathrm{E}}_{n}^{(0)}-4 \widetilde{\mathrm{E}}_{n-1}^{(0)}+3 \widetilde{\mathrm{E}}_{n-2}^{(0)}=0 \quad(\mathrm{n} \geqslant 2)
$$

For $k \geqslant 1$,

$$
\sum_{j=0}^{2 k} \sigma_{j}^{(2 k)} \widetilde{E}_{n-2 k+j}^{(-k)}=0 \quad(n \geqslant 2 k) .
$$

Remark 4.2. For $k=1,2,3$, we have

$$
\begin{aligned}
& \left\{\widetilde{\mathrm{E}}_{\mathfrak{n}}^{(-1)}\right\}_{\mathfrak{n} \geqslant 0}=1,12,109,888,6841,51012,372709,2687088,19200241,136354812, \ldots, \\
& \left\{\widetilde{\mathrm{E}}_{\mathfrak{n}}^{(-2)}\right\}_{\mathfrak{n} \geqslant 0}=1,28,493,7192,95161,1189108,14331493,168625072,1951326961, \ldots, \\
& \left\{\widetilde{\mathrm{E}}_{\mathfrak{n}}^{(-3)}\right\}_{\mathfrak{n} \geqslant 0}=1,60,1837,42840,865081,16022100,280592677,4730230320,77624198641, \ldots,
\end{aligned}
$$

and

$$
\begin{array}{r}
\widetilde{\mathrm{E}}_{n}^{(-1)}-12 \widetilde{\mathrm{E}}_{n-1}^{(-1)}+35 \widetilde{\mathrm{E}}_{n-2}^{(-1)}=0, \\
\widetilde{\mathrm{E}}_{n}^{(-2)}-32 \widetilde{\mathrm{E}}_{n-1}^{(-2)}+374 \widetilde{\mathrm{E}}_{n-2}^{(-2)}-1888 \widetilde{\mathrm{E}}_{n-3}^{(-2)}+3465 \widetilde{\mathrm{E}}_{n-4}^{(-2)}=0, \\
\widetilde{\mathrm{E}}_{n}^{(-3)}-60 \widetilde{\mathrm{E}}_{n-1}^{(-3)}+1465 \widetilde{\mathrm{E}}_{n-2}^{(-3)}-18600 \widetilde{\mathrm{E}}_{n-3}^{(-3)}+129259 \widetilde{\mathrm{E}}_{n-4}^{(-3)}-465180 \widetilde{\mathrm{E}}_{n-5}^{(-3)}+675675 \widetilde{\mathrm{E}}_{n-6}^{(-3)}=0 .
\end{array}
$$

In fact, $\widetilde{\mathrm{E}}_{n}^{(0)}=\left(3^{n+1}-1\right) / 2$ (Cf. [15, A003462]). The sequence $\left\{\widetilde{\mathrm{E}}_{n}^{(-1)}\right\}_{n} \geqslant 0$ is the 6th binomial transform of $0,1,0,1,0,1, \ldots$ ([15, A081200]).

The proof of Theorem 4.1 depends on the following Lemma.

Lemma 4.3. Put

$$
V_{n}:=\left|\begin{array}{cccc}
1 & x_{1} & \cdots & x_{1}^{n-1} \\
1 & x_{2} & \cdots & x_{2}^{n-1} \\
\vdots & \vdots & & \vdots \\
1 & x_{n} & \cdots & x_{n}^{n-1}
\end{array}\right|
$$

and let

$$
V_{n, j}:=\left|\begin{array}{cccccccc}
1 & x_{1} & \cdots & x_{1}^{j-2} & x_{1}^{n} & x_{1}^{j} & \cdots & x_{1}^{n-1} \\
1 & x_{2} & \cdots & x_{2}^{j-2} & x_{1}^{n} & x_{2}^{j} & \cdots & x_{2}^{n-1} \\
\vdots & \vdots & & \vdots & \vdots & \vdots & & \vdots \\
1 & x_{n} & \cdots & x_{n}^{j-2} & x_{n}^{n} & x_{n}^{j} & \cdots & x_{n}^{n-1}
\end{array}\right| \quad(j=1,2, \ldots, n),
$$

where the $j$ th column $x_{1}^{j-1}, x_{2}^{j-1}, \ldots, x_{n}^{j-1}$ of $V_{n}$ is replaced by $x_{1}^{n}, x_{2}^{n}, \ldots, x_{n}^{n}$. Then for $i=1,2, \ldots, n$

$$
v_{n, n-i+1}=(-1)^{i-1}\left(\sum_{1 \leqslant \ell_{1}<\cdots<\ell_{i} \leqslant n} x_{\ell_{1}} \cdots x_{\ell_{i}}\right) v_{n}
$$


Proof. As is well-known, Vandermonde's determinant is given by $V_{n}=\prod_{1 \leqslant i<j \leqslant n}\left(x_{j}-x_{i}\right)$. After the $j$ th column is moved to the $n$th column, we get $V_{n, j}=(-1)^{n-j} W_{n, j}$, where

$$
W_{n, j}=\left|\begin{array}{cccccccc}
1 & x_{1} & \cdots & x_{1}^{j-2} & x_{1}^{j} & \cdots & x_{1}^{n-1} & x_{1}^{n} \\
1 & x_{2} & \cdots & x_{2}^{j-2} & x_{2}^{j} & \cdots & x_{2}^{n-1} & x_{2}^{n} \\
\vdots & \vdots & & \vdots & \vdots & & \vdots & \vdots \\
1 & x_{n} & \cdots & x_{n}^{j-2} & x_{n}^{j} & \cdots & x_{n}^{n-1} & x_{n}^{n}
\end{array}\right|
$$

Then, it is sufficient to prove that

$$
W_{n, j}=\left(\sum_{1 \leqslant \ell_{1}<\cdots<\ell_{n-j+1} \leqslant n} x_{\ell_{1}} \cdots x_{\ell_{n-j+1}}\right) v_{n} .
$$

When $j=1,(4.1)$ is valid because

$$
W_{n, 1}=\left|\begin{array}{cccc}
x_{1} & x_{1}^{2} & \cdots & x_{1}^{n} \\
x_{2} & x_{2}^{2} & \cdots & x_{2}^{n} \\
\vdots & \vdots & & \vdots \\
x_{n} & x_{n}^{2} & \cdots & x_{n}^{n}
\end{array}\right|=x_{1} x_{2} \cdots x_{n} V_{n}
$$

Let $2 \leqslant j \leqslant n-1$. By the column operations from the $j$ th to the second, we have

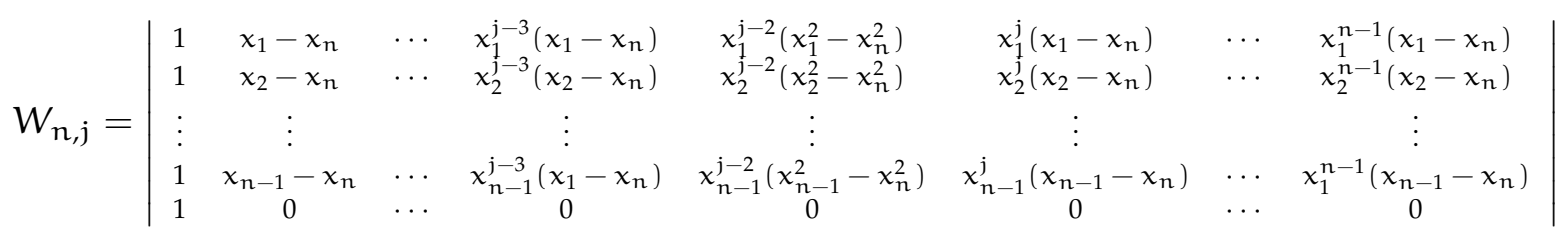

$$
\begin{aligned}
& =(-1)^{n+1} \prod_{\ell=1}^{n-1}\left(x_{\ell}-x_{n}\right) \cdot\left|\begin{array}{ccccccc}
1 & \cdots & x_{1}^{j-3} & x_{1}^{j-2}\left(x_{1}+x_{n}\right) & x_{1}^{j} & \cdots & x_{1}^{n-1} \\
1 & \cdots & x_{2}^{j-3} & x_{2}^{j-2}\left(x_{2}+x_{n}\right) & x_{2}^{j} & \cdots & x_{2}^{n-1} \\
\vdots & & \vdots & \vdots & \vdots & & \vdots \\
1 & \cdots & x_{n-1}^{j-3} & x_{n-1}^{j-2}\left(x_{n-1}+x_{n}\right) & x_{n-1}^{j} & \cdots & x_{n-1}^{n-1}
\end{array}\right| \\
& =\prod_{\ell=1}^{n-1}\left(x_{n}-x_{\ell}\right) \cdot\left(W_{n-1, j-1}+x_{n} W_{n-1, j}\right) \text {. }
\end{aligned}
$$

By induction on $j$ about the relation (4.2), using (4.1) up to $j-1$, we have

$$
\begin{aligned}
W_{n, j} & =\prod_{\ell=1}^{n-1}\left(x_{n}-x_{\ell}\right)\left(\sum_{1 \leqslant \ell_{1}<\cdots<\ell_{n-j+1} \leqslant n-1} x_{\ell_{1}} \cdots x_{\ell_{n-j+1}}\right) v_{n-1}+x_{n} \prod_{\ell=1}^{n-1}\left(x_{n}-x_{\ell}\right) \cdot W_{n-1, j} \\
& =\left(\sum_{1 \leqslant \ell_{1}<\cdots<\ell_{n-j+1} \leqslant n-1} x_{\ell_{1}} \cdots x_{\ell_{n-j+1}}\right) v_{n}+x_{n} \prod_{\ell=1}^{n-1}\left(x_{n}-x_{\ell}\right) \cdot W_{n-1, j} .
\end{aligned}
$$

On the other hand,

$$
W_{n, n}=\left|\begin{array}{ccccc}
1 & x_{1} & \cdots & x_{1}^{n-2} & x_{1}^{n} \\
1 & x_{2} & \cdots & x_{2}^{n-2} & x_{2}^{n} \\
\vdots & \vdots & & \vdots & \vdots \\
1 & x_{n} & \cdots & x_{n}^{n-2} & x_{n}^{n}
\end{array}\right|=x_{n} V_{n}+\prod_{\ell=1}^{n-1}\left(x_{n}-x_{\ell}\right) \cdot W_{n-1, n-1}
$$




$$
\begin{aligned}
= & \left(x_{n-1}+x_{n}\right) V_{n}+\prod_{\ell_{1}=1}^{n-1}\left(x_{n}-x_{\ell_{1}}\right) \prod_{\ell_{2}=1}^{n-2}\left(x_{n-1}-x_{\ell_{2}}\right) \cdot W_{n-2, n-2} \\
= & \left(x_{n}+x_{n-1}+\cdots+x_{3}\right) V_{n} \\
& +\prod_{\ell_{1}=1}^{n-1}\left(x_{n}-x_{\ell_{1}}\right) \prod_{\ell_{2}=1}^{n-2}\left(x_{n-1}-x_{\ell_{2}}\right) \cdots \prod_{\ell_{j-2}=1}^{2}\left(x_{3}-x_{\ell_{j-2}}\right) \cdot W_{2,2} \\
= & \left(x_{1}+x_{2}+x_{3}+\cdots+x_{n-1}+x_{n}\right) V_{n} .
\end{aligned}
$$

Thus, (4.1) is also valid when $j=n$. Now, for $2 \leqslant j \leqslant n-1$, from (4.2) we obtain

$$
\begin{aligned}
W_{n, j}= & \left(\sum_{1 \leqslant \ell_{1}<\cdots<\ell_{n-j+1} \leqslant n-1} x_{\ell_{1}} \cdots x_{\ell_{n-j+1}}\right) v_{n}+x_{n}\left(\sum_{1 \leqslant \ell_{1}<\cdots<\ell_{n-j} \leqslant n-2} x_{\ell_{1}} \cdots x_{\ell_{n-j}}\right) v_{n} \\
& +x_{n} x_{n-1} \prod_{\ell_{1}=1}^{n-1}\left(x_{n}-x_{\ell_{1}}\right) \prod_{\ell_{2}=1}^{n-2}\left(x_{n-1}-x_{\ell_{2}}\right) \cdot W_{n-2, j} \\
= & \left(\sum_{1 \leqslant \ell_{1}<\cdots<\ell_{n-j+1} \leqslant n-1} x_{\ell_{1}} \cdots x_{\ell_{n-j+1}}\right) v_{n}+x_{n}\left(\sum_{1 \leqslant \ell_{1}<\cdots<\ell_{n-j} \leqslant n-2} x_{\ell_{1}} \cdots x_{\ell_{n-j}}\right) v_{n} \\
& \left.+x_{n} x_{n-1}\left(\sum_{1 \leqslant \ell_{1}<\cdots<\ell_{n-j-1} \leqslant n-3} x_{\ell_{1}} \cdots x_{\ell_{n-j-1}}\right) v_{n}+\cdots+x_{n} x_{n-1} \cdots x_{j+2} \sum_{1 \leqslant \ell_{1}<\ell_{2} \leqslant j} x_{\ell_{1}} x_{\ell_{2}}\right) v_{n} \\
& +x_{n} x_{n-1} \cdots x_{j+1} \prod_{\ell_{1}=1}^{n-1}\left(x_{n}-x_{\ell_{1}}\right) \prod_{\ell_{2}=1}^{n-2}\left(x_{n-1}-x_{\ell_{2}}\right) \cdots \prod_{\ell_{n-j}=1}^{j}\left(x_{j+1}-x_{\ell_{n-j}}\right) \cdot W_{j, j} .
\end{aligned}
$$

Since the last term is equal to $x_{n} x_{n-1} \cdots x_{j+1}\left(x_{1}+x_{2}+\cdots+x_{j}\right)$, we get

$$
W_{n, j}=\left(\sum_{1 \leqslant \ell_{1}<\cdots<\ell_{n-j+1} \leqslant n} x_{\ell_{1}} \cdots x_{\ell_{n-j+1}}\right) v_{n},
$$

as desired.

Proof of Theorem 4.1. When $k=0$, by $\widetilde{\mathrm{E}}_{n}^{(0)}=\left(3^{n+1}-1\right) / 2(n \geqslant 0)$, the recurrence relation is clear. Let

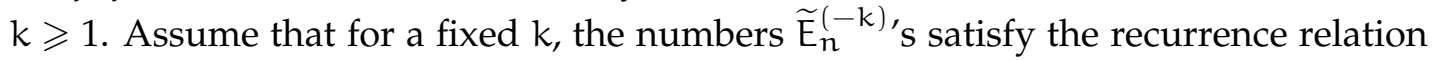

$$
\widetilde{\mathrm{E}}_{n}^{(-\mathrm{k})}+\tau_{\mathrm{k}, 1} \widetilde{\mathrm{E}}_{n-1}^{(-\mathrm{k})}+\tau_{k, 2} \widetilde{\mathrm{E}}_{n-2}^{(-k)}+\cdots+\tau_{k, 2 k} \widetilde{\mathrm{E}}_{n-2 \mathrm{k}}^{(-k)}=0
$$

Since $k$ is fixed, this relation depends only on the part $\left((4 l+3)^{n+1}-(4 l+1)^{n+1}\right)$ in the explicit expression. As $0 \leqslant l \leqslant k$ and $\left\{\begin{array}{l}k \\ 0\end{array}\right\}=0(k \geqslant 1)$, the relation (4.3) is equivalent to the system

$$
(2 \ell+3)^{n+1}+(2 \ell+3)^{n} \tau_{k, 1}+(2 \ell+3)^{n-1} \tau_{k, 2}+\cdots+(2 \ell+3)^{n-2 k+1} \tau_{k, 2 k}=0 \quad(1 \leqslant \ell \leqslant 2 k)
$$

or the system

$$
\begin{array}{r}
5^{2 \mathrm{k}}+5^{2 \mathrm{k}-1} \tau_{\mathrm{k}, 1}+5^{2 \mathrm{k}-2} \tau_{\mathrm{k}, 2}+\cdots+5 \tau_{\mathrm{k}, 2 \mathrm{k}-1}+\tau_{\mathrm{k}, 2 \mathrm{k}}=0, \\
7^{2 \mathrm{k}}+7^{2 \mathrm{k}-1} \tau_{\mathrm{k}, 1}+7^{2 \mathrm{k}-2} \tau_{\mathrm{k}, 2}+\cdots+7 \tau_{\mathrm{k}, 2 \mathrm{k}-1}+\tau_{\mathrm{k}, 2 \mathrm{k}}=0, \\
\vdots \\
(4 \mathrm{k}+3)^{2 \mathrm{k}}+(4 \mathrm{k}+3)^{2 \mathrm{k}-1} \tau_{\mathrm{k}, 1}+(4 \mathrm{k}+3)^{2 \mathrm{k}-2} \tau_{\mathrm{k}, 2}+\cdots+(4 \mathrm{k}+3) \tau_{\mathrm{k}, 2 \mathrm{k}-1}+\tau_{\mathrm{k}, 2 \mathrm{k}}=0 .
\end{array}
$$


Hence, the coefficients $\tau_{k, 1}, \tau_{k, 2}, \ldots, \tau_{k, 2 k}$ are the solution of the matrix equation

$$
\left(\begin{array}{ccccc}
5^{2 k-1} & 5^{2 k-2} & \cdots & 5 & 1 \\
7^{2 k-1} & 7^{2 k-2} & \cdots & 7 & 1 \\
\vdots & \vdots & & \vdots & \vdots \\
(4 k+3)^{2 k-1} & (4 k+3)^{2 k-2} & \cdots & 4 k+3 & 1
\end{array}\right)\left(\begin{array}{c}
\tau_{k, 1} \\
\tau_{k, 2} \\
\vdots \\
\tau_{k, 2 k-1} \\
\tau_{k, 2 k}
\end{array}\right)=\left(\begin{array}{c}
-5^{2 k} \\
-7^{2 k} \\
\vdots \\
-(4 k+1)^{2 k} \\
-(4 k+3)^{2 k}
\end{array}\right) .
$$

The determinant of the $2 \mathrm{k}$ dimensional Vandermonde matrix on the left-hand side is equal to

$$
\mathrm{V}:=\prod_{1 \leqslant \ell_{1}<\ell_{2} \leqslant 2 k}\left(\left(2 \ell_{1}+3\right)-\left(2 \ell_{2}+3\right)\right)=(-2)^{\mathrm{k}(2 \mathrm{k}-1)} \mathrm{V}_{2 \mathrm{k}}
$$

which is the denominator part of $\tau_{k, j}$ by Cramer. By applying Lemma 4.3 with $n=2 \mathrm{~m}$ and $x_{i}=2 i+3$ $(i=1,2, \ldots, 2 k)$, the numerator part of $\tau_{k, j}$ is given by

$$
(-1)^{j}\left(\sum_{1 \leqslant \ell_{1}<\cdots<\ell_{j} \leqslant 2 k}\left(2 \ell_{1}+3\right) \cdots\left(2 \ell_{j}+3\right)\right) \cdot v .
$$

Hence, we have

$$
\tau_{k, j}=(-1)^{j} \sum_{1 \leqslant \ell_{1}<\cdots<\ell_{j} \leqslant 2 k}\left(2 \ell_{1}+3\right) \cdots\left(2 \ell_{j}+3\right)=\sigma_{2 k-j}^{(2 k)} \quad(j=1,2, \ldots, 2 k) .
$$

For an integer $k$, poly-Euler numbers of the second kind $\widehat{E}_{n}^{(k)}(n \geqslant 0)$ are defined by $([10,11])$

$$
\frac{\operatorname{Li}_{k}\left(1-e^{-4 x}\right)}{4 \sinh x}=\sum_{n=0}^{\infty} \widehat{E}_{n}^{(k)} \frac{x^{n}}{n !}
$$

When $k=1, \widehat{E}_{n}=\widehat{E}_{n}^{(1)}$ are the original Euler numbers of the second kind (or complimentary Euler numbers), defined by

$$
\frac{x}{\sinh x}=\sum_{n=0}^{\infty} \widehat{E}_{n} \frac{x^{n}}{n !} .
$$

Poly-Euler numbers of the second kind can be expressed in terms of poly-Bernoulli numbers ([10, Theorem 3.1], [11, Lemma 3.1]):

$$
\widehat{E}_{n}^{(k)}=\frac{1}{2} \sum_{m=0}^{n}\left(\begin{array}{l}
n \\
m
\end{array}\right) 4^{m}\left((-1)^{n-m}-(-3)^{n-m}\right) B_{m}^{(k)} .
$$

In the case of negative index, poly-Euler numbers of the second kind have an explicit expression ([10, Theorem 3.3], [11, Lemma 3.2]):

$$
\widehat{E}_{n}^{(-k)}=\frac{(-1)^{k}}{2} \sum_{l=0}^{k}(-1)^{l} l !\left\{\begin{array}{l}
k \\
l
\end{array}\right\}\left((4 l+3)^{n}+(4 l+1)^{n}\right) \quad(k \geqslant 0) .
$$

Notice that $E_{n}^{(-k)}$ are all integers. Euler numbers of the second kind with negative indices satisfy the same recurrence formula as those in Theorem 4.1. 
Theorem 4.4. For $\mathrm{k}=0$,

$$
\widehat{\mathrm{E}}_{n}^{(0)}-4 \widehat{\mathrm{E}}_{n-1}^{(-k)}+3 \widehat{\mathrm{E}}_{n-2}^{(-k)}=0 \quad(\mathrm{n} \geqslant 2)
$$

For $k \geqslant 1$,

$$
\sum_{j=0}^{2 k} \sigma_{j}^{(2 k)} \widehat{E}_{n-2 k+j}^{(-k)}=0 \quad(n \geqslant 2 k) .
$$

Remark 4.5. For $k=0,1,2,3$, we have

$$
\begin{aligned}
&\left\{\widehat{\mathrm{E}}_{\mathfrak{n}}^{(0)}\right\}_{\mathfrak{n}} \geqslant 0=1,2,5,14,41,122,365,1094,3281,9842,29525,88574, \ldots, \\
&\left\{\widehat{\mathrm{E}}_{\mathfrak{n}}^{(-1)}\right\}_{\mathfrak{n}} \geqslant 0=1,6,37,234,1513,9966,66637,450834,3077713,21153366, \ldots, \\
&\left\{\widehat{\mathrm{E}}_{\mathfrak{n}}^{(-2)}\right\}_{\mathfrak{n}} \geqslant 0=1,14,165,1826,19689,210134,2236365,23819306,254327889, \ldots, \\
&\left\{\widehat{\mathrm{E}}_{\mathfrak{n}}^{(-3)}\right\}_{\mathfrak{n} \geqslant 0}=1,30,613,10770,175465,2741670,41809933,628464090,9366724945, \ldots
\end{aligned}
$$

In fact, $\widehat{\mathrm{E}}_{\mathfrak{n}}^{(0)}=\left(3^{n}+1\right) / 2([15, \mathrm{~A} 007051])$. The sequence $\left\{\widehat{\mathrm{E}}_{\mathfrak{n}}^{(-1)}\right\}_{\mathfrak{n} \geqslant 0}$ is the 6th binomial transform of $1,0,1,0,1, \ldots([15, \mathrm{~A} 081188])$.

Proof of Theorem 4.4. The proof can be done as the same as that of Theorem 4.1. However, we shall prove the similar result by a different method, which is also applicable to that of Theorem 4.1. Since

$$
x(x-1) \cdots(x-n+1)=\sum_{\ell=0}^{n}(-1)^{n-\ell}\left[\begin{array}{l}
n \\
\ell
\end{array}\right] x^{\ell},
$$

we get

$$
\begin{aligned}
(x-5 \mid 2)_{n}=\sum_{\ell=0}^{n}(-2)^{n-\ell}\left[\begin{array}{l}
n \\
\ell
\end{array}\right](x-5)^{\ell} & =\sum_{\ell=0}^{n}(-2)^{n-\ell}\left[\begin{array}{l}
n \\
\ell
\end{array}\right] \sum_{j=0}^{\ell}\left(\begin{array}{l}
\ell \\
j
\end{array}\right)(-5)^{\ell-j} x^{j} \\
& =\sum_{j=0}^{n} \sum_{\ell=j}^{n}(-2)^{n-\ell}(-5)^{\ell-j}\left(\begin{array}{l}
\ell \\
j
\end{array}\right)\left[\begin{array}{l}
n \\
\ell
\end{array}\right] x^{j} .
\end{aligned}
$$

Hence, for $j=0,1, \ldots, n$, we have

$$
\sigma_{j}^{(n)}=\sum_{\ell=j}^{n}(-2)^{n-\ell}(-5)^{\ell-j}\left(\begin{array}{l}
\ell \\
j
\end{array}\right)\left[\begin{array}{l}
n \\
\ell
\end{array}\right]
$$

Therefore, for $k \geqslant 1$ and $n \geqslant 2 k$,

$$
\begin{aligned}
& \sum_{j=0}^{2 k} \sigma_{j}^{(2 k)} \widehat{E}_{n-2 k+j}^{(-k)} \\
& \quad=\sum_{j=0}^{2 k} \sum_{\ell=j}^{2 k}(-2)^{2 k-\ell}(-5)^{\ell-j}\left(\begin{array}{l}
\ell \\
j
\end{array}\right)\left[\begin{array}{c}
2 k \\
\ell
\end{array}\right] \frac{(-1)^{k}}{2} \sum_{r=0}^{k}(-1)^{r} r !\left\{\begin{array}{l}
k \\
r
\end{array}\right\}\left((4 r+3)^{n-2 k+j}+(4 r+1)^{n-2 k+j}\right) .
\end{aligned}
$$

Now

$$
\sum_{j=0}^{2 k} \sum_{\ell=j}^{2 k}(-2)^{2 k-\ell}(-5)^{\ell-j}\left(\begin{array}{l}
\ell \\
j
\end{array}\right)\left[\begin{array}{c}
2 k \\
\ell
\end{array}\right] \frac{(-1)^{k}}{2} \sum_{r=0}^{k}(-1)^{r} r !\left\{\begin{array}{l}
k \\
r
\end{array}\right\}(4 r+3)^{n-2 k+j}
$$




$$
\begin{aligned}
& =2^{2 k} \sum_{r=0}^{k}(-1)^{r} r !\left\{\begin{array}{l}
k \\
r
\end{array}\right\}(4 r+3)^{n-2 k} \sum_{\ell=0}^{2 k}\left(\frac{5}{2}\right)^{\ell}\left[\begin{array}{c}
2 k \\
\ell
\end{array}\right] \sum_{j=0}^{\ell}\left(\begin{array}{l}
\ell \\
j
\end{array}\right)\left(-\frac{4 r+3}{5}\right)^{j} \\
& =2^{2 k} \sum_{r=0}^{k}(-1)^{r} r !\left\{\begin{array}{l}
k \\
r
\end{array}\right\}(4 r+3)^{n-2 k} \sum_{\ell=0}^{2 k}\left(\frac{5}{2}\right)^{\ell}\left[\begin{array}{c}
2 k \\
\ell
\end{array}\right]\left(1-\frac{4 r+3}{5}\right)^{\ell} \\
& =2^{2 k} \sum_{r=0}^{k}(-1)^{r} r !\left\{\begin{array}{l}
k \\
r
\end{array}\right\}(4 r+3)^{n-2 k} \sum_{\ell=0}^{2 k}\left[\begin{array}{c}
2 k \\
\ell
\end{array}\right](1-2 r)^{\ell} \\
& =2^{2 k} \sum_{r=0}^{k}(-1)^{r} r !\left\{\begin{array}{l}
k \\
r
\end{array}\right\}(4 r+3)^{n-2 k}(2 r-1)(2 r-2) \cdots(2 r-2 k),
\end{aligned}
$$

which is equal to 0 because $(2 r-1)(2 r-2) \cdots(2 r-2 k)=0$ for $r=1,2, \ldots, k$ and $\left\{\begin{array}{l}k \\ 0\end{array}\right\}=0$ for $k \geqslant 1$. Similarly,

$$
\begin{gathered}
\sum_{j=0}^{2 k} \sum_{\ell=j}^{2 k}(-2)^{2 k-\ell}(-5)^{\ell-j}\left(\begin{array}{l}
\ell \\
j
\end{array}\right)\left[\begin{array}{c}
2 k \\
\ell
\end{array}\right] \frac{(-1)^{k}}{2} \sum_{r=0}^{k}(-1)^{r} r !\left\{\begin{array}{l}
k \\
r
\end{array}\right\}(4 r+1)^{n-2 k+j} \\
=2^{2 k} \sum_{r=0}^{k}(-1)^{r} r !\left\{\begin{array}{l}
k \\
r
\end{array}\right\}(4 r+1)^{n-2 k}(2 r-2)(2 r-3) \cdots(2 r-2 k-1),
\end{gathered}
$$

which is equal to 0 because $(2 r-2)(2 r-3) \cdots(2 r-2 k-1)=0$ for $r=1,2, \ldots, k$ and $\left\{\begin{array}{l}k \\ 0\end{array}\right\}=0$ for $k \geqslant 1$.

\section{Leaping relations}

Since the numbers $c_{n}^{(-2)}$ satisfy the three-term recurrence relation $c_{n}^{(-2)}+(2 n-5) c_{n-1}^{(-2)}+(n-3)^{2} c_{n-2}^{(-2)}$ for $n \geqslant 4$, they also can satisfy the leaping recurrence relations of the form

$$
A(n) c_{n}^{(-2)}+B(n) c_{n-r}^{(-2)}+C(n) c_{n-2 r}^{(-2)}=0 \quad(n \geqslant 2 r+2)
$$

for a positive integer $r$.

Given a three-term recurrence formula $Z_{n}=T(n) Z_{n-1}+U(n) Z_{n-2}(n \geqslant 2)$ with arbitrary initial values $Z_{0}$ and $Z_{1}$, and two sequence of integers are eventually quasi-periodic as

$$
\begin{aligned}
& \{\mathrm{T}(\mathrm{n})\}_{n} \geqslant 0=\mathrm{a}_{0}, \mathrm{a}_{1} \ldots, \mathrm{a}_{\rho}, \overline{\mathrm{T}_{1}(\mathrm{k}), \mathrm{T}_{2}(\mathrm{k}), \ldots, \mathrm{T}_{w}(\mathrm{k})_{\mathrm{k}=1}^{\infty},} \\
& \{\mathrm{U}(\mathrm{n})\}_{n \geqslant 0}=\mathrm{b}_{0}, \mathrm{~b}_{1} \ldots, \mathrm{b}_{\rho}, \overline{\mathrm{U}_{1}(\mathrm{k}), \mathrm{U}_{2}(\mathrm{k}), \ldots, \mathrm{U}_{w}(\mathrm{k})_{\mathrm{k}=1}^{\infty} .}
\end{aligned}
$$

For integers $a$ and $l$ with $l \geqslant 1$, we define the determinant

$$
\mathrm{K}_{\mathrm{l}}(\mathrm{a})=\left|\begin{array}{cccccc}
\mathrm{T}(\mathrm{a}) & 1 & 0 & & & \\
-\mathrm{U}(\mathrm{a}+1) & \mathrm{T}(\mathrm{a}+1) & 1 & & & \\
0 & -\mathrm{U}(\mathrm{a}+2) & \mathrm{T}(\mathrm{a}+2) & & & 0 \\
& & & \ddots & & 1 \\
& & & & \mathrm{~T}(\mathrm{a}+\mathrm{l}-2) & -\mathrm{U}(\mathrm{a}+\mathrm{l}-1) \\
& & & 0 & \mathrm{~T}(\mathrm{a}+\mathrm{l}-1)
\end{array}\right|
$$

with $K_{0}(a)=1$. Let $\Omega(M)=U(M-r) U(M-r+1) \cdots U(M-1)$ with $M=(n-1) r+i+2$. Then we have the following ([4, Theorem 2]). 
Lemma 5.1. For any integers $r$ and $i$ with $r \geqslant 2,0 \leqslant \rho \leqslant i<\rho+r$, and $n \geqslant 2$,

$$
\begin{aligned}
& K_{r-1}(M-r) \cdot Z_{r n+i}-\left(K_{r-1}(M) K_{r}(M-r)+U(M) K_{r-1}(M-r) K_{r-2}(M+1)\right) \cdot Z_{r n-r+i} \\
& \quad+(-1)^{r} \Omega(M) K_{r-1}(M) \cdot Z_{r n-2 r+i}=0 .
\end{aligned}
$$

We apply Lemma 5.1 as $T(n)=-(2 n-5)$ and $U(n)=-(n-3)^{2}$. When $r=2$ and $i=0$, we get $M=2 n, \Omega(M)=(2 n-4)^{2}(2 n-5)^{2}, K_{1}(M)=-(4 n-5), K_{1}(M-r)=-(4 n-9)$, and $K_{2}(M-r)=$ $12 n^{2}-48 n+47$. Thus, we have

$$
-(4 n-9) \mathbf{c}_{n}^{(-2)}+2(4 n-7)\left(4 n^{2}-14 n+11\right) c_{n-2}^{(-2)}-(2 n-4)^{2}(2 n-5)^{2}(4 n-5) c_{n-4}^{(-2)} .
$$

When $r=2$ and $i=1$, we get $M=2 n+1, \Omega(M)=(2 n-3)^{2}(2 n-4)^{2}, K_{1}(M)=-(4 n-3), K_{1}(M-r)=$ $-(4 n-7)$, and $K_{2}(M-r)=12 n^{2}-36 n+26$. Thus, we have

$$
-(4 n-7) c_{2 n+1}^{(-2)}+2(4 n-5)\left(4 n^{2}-10 n+5\right) c_{2 n-1}^{(-2)}-(2 n-3)^{2}(2 n-4)^{2}(4 n-3) c_{2 n-3}^{(-2)} .
$$

Proposition 5.2. For $\mathrm{n} \geqslant 3$,

$$
\begin{gathered}
(4 n-9) c_{2 n}^{(-2)}-2(4 n-7)\left(4 n^{2}-14 n+11\right) c_{2 n-2}^{(-2)}+(2 n-3)^{2}(2 n-4)^{2}(4 n-5) c_{2 n-4}^{(-2)}=0, \\
(4 n-7) c_{2 n+1}^{(-2)}-2(4 n-5)\left(4 n^{2}-10 n+5\right) c_{2 n-1}^{(-2)}+(2 n-3)^{2}(2 n-4)^{2}(4 n-3) c_{2 n-3}^{(-2)}=0 .
\end{gathered}
$$

For example,

$$
\begin{aligned}
3 c_{6}^{(-2)}-50 c_{4}^{(-2)}+28 c_{2}^{(-2)} & =0, \\
5 c_{7}^{(-2)}-154 c_{5}^{(-2)}+324 c_{3}^{(-2)} & =0, \\
7 c_{8}^{(-2)}-342 c_{6}^{(-2)}+1584 c_{4}^{(-2)} & =0, \\
9 c_{9}^{(-2)}-638 c_{7}^{(-2)}+5200 c_{5}^{(-2)} & =0 .
\end{aligned}
$$

It is similarly shown for the cases when $r \geqslant 3$, but the expressions become more complicated.

For the three-term recurrence relation of Bernoulli numbers $B_{n}^{(-2)}-5 B_{n-1}^{(-2)}+6 B_{n-2}^{(-2)}$, leaping recurrence relations are much simpler. When $r=2$, we have $5 B_{n}^{(-2)}-65 B_{n-2}^{(-2)}+180 B_{n-4}^{(-2)}=0$ or

$$
\mathrm{B}_{\mathrm{n}}^{(-2)}-13 \mathrm{~B}_{\mathrm{n}-2}^{(-2)}+36 \mathrm{~B}_{\mathrm{n}-4}^{(-2)}=0 \text {. }
$$

In fact, we can get a more general case by using the following result ([8, Theorem 1]).

Lemma 5.3. If the sequence $\left\{Z_{n}\right\}_{n}$ satisfies the three-term relation $Z_{n}=a_{1} Z_{n-1}+a_{2} Z_{n-2}$, then for any positive integer $\mathrm{r}$ we have

$$
Z_{n}=r \sum_{i=0}^{\lfloor r / 2\rfloor} \frac{(r-i-1) !}{i !(r-2 i) !} a_{1}^{r-2 i} a_{2}^{i} \cdot Z_{n-r}-(-1)^{r} a_{2}^{r} \cdot Z_{n-2 r} \quad(n \geqslant 2 r) .
$$

By applying Lemma 5.3 as $a_{1}=5$ and $a_{2}=-6$, we have the following.

\section{Proposition 5.4.}

$$
\mathrm{B}_{\mathrm{n}}^{(-2)}=\mathrm{r} \sum_{i=0}^{\lfloor\mathrm{r} / 2\rfloor} \frac{(\mathrm{r}-\mathrm{i}-1) !}{i !(r-2 i) !} 5^{r-2 i}(-6)^{i} \cdot B_{n-r}^{(-2)}-6^{r} \cdot B_{n-2 r}^{(-2)} \quad(n \geqslant 2 r)
$$


Concerning the sequence $\left\{c_{n}^{(-3)}\right\}$, a four-term recurrence relation

$$
c_{n}^{(-3)}+3(n-3) c_{n-1}^{(-3)}+\left(3 n^{2}-21 n+37\right) c_{n-2}^{(-3)}+(n-4)^{3} c_{n-3}^{(-3)} \quad(n \geqslant 5)
$$

holds. However, no general formula for leaping relation has been found. As for Bernoulli numbers $B_{\mathfrak{n}}^{(-3)}$, a four-term relation

$$
\mathrm{B}_{\mathrm{n}}^{(-3)}-9 \mathrm{~B}_{n-1}^{(-3)}+26 \mathrm{~B}_{\mathrm{n}-2}^{(-3)}-24 \mathrm{~B}_{n-3}^{(-3)}=0
$$

has only constant coefficients, by applying [8, Theorem 2], we have a leaping relation

$$
\begin{aligned}
B_{n}^{(-3)}= & r \sum_{j=0}^{\lfloor r / 3\rfloor} \sum_{i=0}^{(r-3 j) / 2} \frac{(r-i-2 j-1) !}{i ! j !(r-2 i-3 j) !} 9^{r-2 i-3 j}(-26)^{i} \cdot 24^{j} B_{n-r}^{(-3)} \\
& -r \sum_{j=0}^{\lfloor r / 3\rfloor} \sum_{i=0}^{\lfloor(r-3 j) / 2\rfloor}(-1)^{r+i+j} \frac{(r-i-2 j-1) !}{i ! j !(r-2 i-3 j) !} 9^{i}(-26)^{r-2 i-3 j} \cdot 24^{i+2 j} \cdot B_{n-2 r}^{(-3)}+24^{r} B_{n-3 r}^{(-3)} \quad(n>3 r) .
\end{aligned}
$$

\section{Comments}

Sasaki [14] announced more recurrence formulas of poly-Bernoulli numbers from the duality theorem $\mathrm{B}_{\mathrm{n}}^{(-\mathrm{k})}=\mathrm{B}_{\mathrm{k}}^{(-\mathrm{n})}$, and combinatorial interpretations about some relations. However, poly-Cauchy numbers and poly-Euler numbers do not satisfy the duality theorem. Any combinatorial interpretation of polyCauchy numbers has not been known yet, though a few are discovered about poly-Euler numbers [13].

\section{Acknowledgment}

The author thanks the anonymous referee for the detailed comments and suggestions, which improved the quality of the paper.

\section{References}

[1] T. Arakawa, T. Ibukiyama, M. Kaneko, Bernoulli numbers and zeta functions. With an appendix by Don Zagier, Springer, Tokyo, (2014). 2

[2] T. Arakawa, M. Kaneko, On Poly-Bernoulli numbers, Comment. Math. Univ. St. Paul., 48 (1999), 159-167. 1

[3] L. Comtet, Advanced Combinatorics, D. Reidel Publishing Co., Dordrecht, (1974). 1

[4] C. Elsner, T. Komatsu, On the residue classes of integer sequences satisfying a linear three-term recurrence formula, Linear Algebra Appl., 429 (2008), 933-947. 5

[5] K. Kamano, T. Komatsu, Poly-Cauchy polynomials, Mosc. J. Comb. Number Theory, 3 (2013), 61-87. 1, 3

[6] K. Kamano, T. Komatsu, Some congruence relations of poly-Cauchy numbers, J. Comb. Number Theory, 5 (2014), 145-150. 1

[7] M. Kaneko, Poly-Bernoulli numbers, J. Théor. Nombres Bordeaux, 9 (1997), 221-228. 1

[8] T. Komatsu, Four-term leaping recurrence relations, Linear Multilinear Algebra, 58 (2010), 875-886. 5, 5

[9] T. Komatsu, Poly-Cauchy numbers, Kyushu J. Math., 67 (2013), 143-153. 1, 1, 3

[10] T. Komatsu, Complementary Euler numbers, Period. Math. Hungar., 75 (2017), 302-314. 4

[11] T. Komatsu, On poly-Euler numbers of the second kind, arXiv, 2018 (2018), 16 pages. 4

[12] D. Merlini, R. Sprugnoli, M. C. Verri, The Cauchy numbers, Discrete Math., 306 (2006), 1906-1920. 1

[13] Y. Ohno, Y. Sasaki, On poly-Euler numbers, J. Aust. Math. Soc., 103 (2017), 126-144. 4, 6

[14] Y. Sasaki, Recurrence formulas for poly-Bernoulli numbers and their combinatoric interpretations, Presented at the workshop of Analytic Number Theory and Related Topics (Research Institute for Mathematical Sciences, Kyoto University), 2018 (2018), 11 pages. 1, 6

[15] N. J. A. Sloane, The on-line encyclopedia of integer sequences, Notices Amer. Math. Soc., 65 (2018), 1062-1074. 1, 1, $4.2,4.5$ 